\title{
An Attempt to model the Salam-Weinberg Higgs ${ }^{1}$
}

\author{
H. Schlereth \\ Paul Scherrer Institute \\ CH-5232 Villigen-PSI, Switzerland \\ and \\ Institute for Theoretical Physics \\ University of Zürich, Switzerland
}

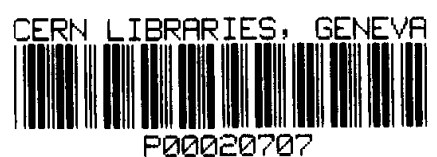

\footnotetext{
${ }^{1}$ Partially supported by "Schweizerischer Nationalfonds"
} 


\title{
An Attempt to model the Salam-Weinberg Higgs ${ }^{1}$
}

\author{
H. Schlereth \\ Paul Scherrer Institute \\ CH-5232 Villigen-PSI, Switzerland \\ and \\ Institute for Theoretical Physics \\ University of Zürich, Switzerland
}

\begin{abstract}
It is shown that in a two scale confining substructure model for Standard Model fermions and weak bosons respectively where the two scales obey $\Lambda^{\prime} \gg \Lambda$ a sea-saw scale $\mu \ll$ $\Lambda, \Lambda^{\prime}\left(\mu=\Lambda \cdot\left(\frac{\Lambda}{\Lambda^{\prime}}\right)\right)$ is generated. We discuss the possible existence of a critical value $\mu_{c}$ of $\mu$ where, due to an effect of level repulsion, the low energy part of the effective bound state theory lives at the Higgs-confinement phase boundary. Then $\mu_{c}$ is identified with the weak scale. We present correlators to be measured at the phase boundary in a lattice definition of the model to determine the mass ratio $m_{\text {Higgs }} / M_{W}$. We argue that by the Weinberg-Witten theorem only the Higgs phase is accessible and a light Higgs exists at the phase boundary. An argument that its effective renormalized couplings are weak is given.
\end{abstract}

\footnotetext{
${ }^{1}$ Partially supported by "Schweizerischer Nationalfonds"
} 
The breaking of the $S U(2) \times U(1)$ group in the Standard Model [S.M] [1] incorporated in the Higgs sector is, without doubt, the basic problem in present high energy physics. Naturally any attempt to understand this phenomenon has to go beyond the S.M. itself.

Present attempts center around supersymmetric [SUSY] extensions of the S.M. [2] and technicolor type theories [TC] [3].

In the former the Higgs is embedded as an elementary scalar in a larger boson-fermion symmetry which makes it easier to understand the (eventual) presence of a light Higgs.

In TC theories the existence of a new strongly interacting symmetry breaking sector (basically modelled as a scaled up QCD) is assumed. These models are typically discussed in terms of chiral Lagrangians [4] and naturally contain a heavy Higgs.

In the present note we address the question whether the Higgs might arise as a collective excitation in the infrared [IR] of a confining subconstituent theory with confinement scale in the $\mathrm{O}(1) \mathrm{TeV}$ region. We first have to explain what we mean by IR.

We choose a confining theory whose lightest bound state has the quantum numbers of the weak boson. This model was first proposed in $[5]^{1}$ and is numerically further investigated in [6]. The main point of [5] is that with Weyl instead of Dirac fermions one can construct a two flavour confining theory without unwanted Goldstone bosons.

The continuum Lagrangian is simply

$$
\mathcal{L}=\frac{1}{g^{2}} \cdot \operatorname{tr}\left(F_{\mu \nu} F^{\mu \nu}\right)+\chi \dagger \not D \chi
$$

where $F_{\mu \nu}$ is an SU(2) Yang-Mills field strength, $\not D$ the gauge covariant derivative and $\chi$ a Weyl spinor that transforms in the fundamental representation of both the gauged SU(2) and a global SU(2) of isospin [5].

The lowest bound states are given by matrix elements of the bilinear operators

$$
\begin{aligned}
W_{\mu}^{A}(x) & \equiv \chi^{\dagger}(x) \sigma_{\mu} T^{A} \chi(x) \\
W_{\mu}(x) & \equiv \chi^{\dagger}(x) \sigma_{\mu} \chi(x) \\
S(x) & \equiv \chi(x) Q \chi(x)-\chi^{\dagger}(x) Q \chi^{\dagger}(x)
\end{aligned}
$$

where $\left(\sigma^{\mu}\right) \equiv(i, \vec{\sigma})$ (in Minkowski space), $\left(T^{A}\right) \equiv\left(T^{1}, T^{2}, T^{3}\right)$ are the Pauli matrices of isospin and $Q \equiv i \sigma_{2} i T_{2} i \tau_{2}$ with $i \tau_{2}$ denoting the antisymmetrization in the indices of the gauged SU(2).

Preliminary results from a lattice simulation of (1) (a Majorana mass term has been omitted in (1)) [6] are consistent with the vector-isotriplet $W_{\mu}^{A}(x)$ being the lightest bound state. If this is normalized to $m_{W} \cong 80 \mathrm{GeV}$ the masses of the vector isosinglet and the scalar turn out to be in the $100 \mathrm{GeV}$ region. We will however not use this normalization but

\footnotetext{
${ }^{1}$ In [5] an unfortunate sign error has entered the calculations due to a wrong factor $\underline{i}$ in the fourth and fifth term of equation (3). To interpret the results correctly one has to interchange isosinglet $\leftrightarrow$ isovector.
} 
instead assume a confinement scale of $\Lambda \cong O(1 \mathrm{TeV})$ because in this energy region the take over of "new physics" is expected [7].

To proceed let us assume that there is a second layer of substructure (of composite quarks, say) at a scale $\Lambda^{\prime} \gg \Lambda$. One may think about this second layer along the lines of [8], but in fact we need not specify it in any great detail here.

For the present purpose it is enough to assume the presence of one heavy $\left(M \cong O\left(\Lambda^{\prime}\right)\right)$ isodoublet bound state fermion $T(x)$ whose isospin current forms at energies much below $\Lambda^{\prime}$ a residual four fermion interaction with the isospin current of (1) at the scale $\Lambda$. If we further include a selfinteraction of $T(x)$ at the scale $\Lambda^{\prime}$ and the heavy fermion mass term we can write

$$
\Delta \mathcal{L}=\frac{1}{\Lambda^{2}} \cdot \chi^{\dagger} \sigma_{\mu} T^{A} \chi \cdot \bar{T} \gamma^{\mu} T^{A} T+\frac{1}{\Lambda^{2}} \cdot \bar{T} \gamma_{\mu} T^{A} T \cdot \bar{T} \gamma^{\mu} T^{A} T-M \bar{T} T
$$

such that the theory we are going to study has the partition function

$$
Z=\int d \bar{T} d T d \chi^{\dagger} d \chi d A_{\mu} e^{-(\mathcal{L}+\Delta \mathcal{L})}
$$

where $A_{\mu}(x)$ is the $\mathrm{SU}(2)$ gauge potential contained in (1).

In (3) the dimensionless constants in front of the four fermion interactions are normalized to one. Since $M \cong O\left(\Lambda^{\prime}\right)$ and we are at energy scales $\ll \Lambda^{\prime}$ the kinetic term of the $T(x)$ fermion was neglected in (3) against the mass term ${ }^{2}$. Further we have written only the terms in the spin, isospin one channel which is supposed to mix with the theory (1).

It is possible to contemplate substructure models [8] where (3) is plausibel with no mixing in the isospin one channel ${ }^{3}$ of (2). Then $\Lambda$ in the first term of (3) is not necessarily the confinement scale of (1) but some effective scale smaller than $\Lambda^{\prime}$ generated by critical fermionic screening of the strong gauge forces [8]. At a later stage it should be possible to derive (3) from such type of models. But for the rest of this paper we take (3) for granted with $\Lambda \ll \Lambda^{\prime}$.

Next we change the integration over the heavy fermion in (4) to integrating over the bilinear variable

$$
V_{\mu}^{A}(x)=\frac{1}{\Lambda^{2}} \cdot \bar{T}(x) \gamma_{\mu} T^{A} T(x)
$$

This is done by inserting

$$
1=\prod_{x} \int d V_{\mu}^{A}(x) \delta\left(\frac{1}{\Lambda^{2}} \bar{T}(x) \gamma_{\mu} T^{A} T(x)-V_{\mu}^{A}(x)\right)
$$

\footnotetext{
${ }^{2}$ At energy $E \ll \Lambda^{\prime}$ the $T(x)$ particle is "produced" far off-shell with a lifetime $T \sim \frac{1}{\Lambda^{\prime}}$.

${ }^{3}$ A potential mixing in the scalar sector would lead to a scalar with mass $\approx O\left(M_{W}\right)$ as we shall discuss. Since in a model as in [8],[5] this scalar couples only to lefthanded fermions and most likely cannot decay into $W / Z$ for kinematic reasons it remains experimentally well hidden.
} 
into (4) and using the integral representation of the $\delta$-function. Further it is assumed that fluctuations in the functional integral are small on the scale $\Lambda^{\prime}$ so that when integrating out $T(x)$ the quadratic approximation to the $\operatorname{tr} \log$-term is enough. Finally a coarse grained space-time integration in terms of hypercubes of size $\left(\frac{1}{\Lambda^{\prime}}\right)^{4}$ is defined

$$
\int d^{4} x \rightarrow \sum_{x} \frac{1}{\Lambda^{\prime 4}}
$$

Then (4) takes the form

$$
Z=\int d V_{\mu}^{A} d \chi^{\dagger} d \chi d A_{\mu} e^{-\int d^{4} x\left[\mathcal{L}+\chi^{\dagger} \sigma^{\mu} T^{A} \chi \cdot V_{\mu}^{A}+\frac{\mu^{2}}{4} \cdot V_{\mu}^{A^{2}}\right]}
$$

with the sea-saw type mass scale

$$
\mu=O\left[\Lambda \cdot\left(\frac{\Lambda}{\Lambda^{\prime}}\right)\right]
$$

For instance $\Lambda=1 \mathrm{TeV}$ and $\Lambda^{\prime}=10 \mathrm{TeV}$ gives $\mu=O(100 \mathrm{GeV})$. It is the energy region $\approx O(\mu)$ which we call IR in this note. It is the aim of the present project to explore the hierarchy $\mu \ll \Lambda, \Lambda^{\prime}$.

Since the scale $\mu$ is tied to $V_{\mu}^{A}(x)$ this variable should be integrated out in the "hadronized" phase and not in the perturbative one.

We first apply heuristic methods known from low energy QCD [9] to (1) to take into account confinement.

The method consists of integrating out the gluons by summing all one-gluon irreducible Greenfunctions [OGIG] and adding confinement inspired assumptions. Basically it is assumed that the OGIG are constant at distances large compared to the confinement scale. After introducing collective bilinear fields through suitable $\delta$-function identities and performing a derivative expansion in the relative coordinates one winds up for the partition function $\hat{Z}$ of (1) with

$$
\hat{Z}=\int d \chi^{\dagger} d \chi d N e^{+\int d^{4} x\left[\chi^{\dagger} \not \partial \chi-\chi^{\dagger} N \chi-\frac{m^{2}}{4} \operatorname{tr} N^{2}\right]}
$$

with $m^{2}$ a mass parameter and the collective field $N(x)=\sigma^{\mu} \cdot W_{\mu}(x)+\sigma^{\mu} T^{A} W_{\mu}^{A}(x)$.

(9) is in the approximation which corresponds to the Nambu-Lasinio form [9]. For simplicity we have in (9) not treated the scalar $S$ defined in (2). In (9) only fields that vary slowly on the scale $4 \pi \Lambda \cong O(10 \mathrm{TeV})$ are included. Gauge fixing terms are not denoted explicitely.

It is reasonable to expect that the assumptions made to get (9) starting from (1) remain valid also if the system is in contact with the second layer. Then combination of (7) with 
(9) leads to

$$
Z=\int d V_{\mu}^{A} d W_{\mu}^{A} d \chi^{\dagger} d \chi e^{+\int d^{4} x\left[\chi^{\dagger}(\not \partial+W+V) \chi-\frac{m_{W}^{2}}{4} W_{\mu}^{A^{2}}-\frac{\mu^{2}}{4} V_{\mu}^{A^{2}}\right]}
$$

with $W=\sigma^{\mu} T^{A} W_{\mu}^{A}$ etc. and we have explicitely written only the vector isotriplet contribution.

Next we integrate out the fermions (to get the "hadronized" form) and keep only the first term in the derivative expansion of the determinant. Finally integrating over $V_{\mu}^{A}$ after the substitution $W \rightarrow W+V$ we get [9].

$$
Z=\int d W_{\mu}^{A} e^{-\int d^{4} x\left[\frac{1}{g^{2}} \cdot \operatorname{tr} W_{\mu \nu}^{2}+\frac{M_{W}^{2}}{4} \operatorname{tr} W_{\mu}^{2}\right]}
$$

$W_{\mu \nu}$ is the nonabelian field strength of $W_{\mu}, g$ a coupling constant and

$$
M_{W}^{2}=m_{W}^{2} \cdot\left(1-\frac{1}{1+\frac{\mu^{2}}{m_{W}^{2}}}\right)
$$

Although (12) has been arrived at from $(1,7)$ after a number of crude approximations it has physical properties which should hold true more generally.

It is always $M_{W}^{2} \geq 0$ so that no spontaneous breaking of Lorentzinvariance is induced. $M_{W}^{2}=0$ is obtained for $\mu^{2}=0$ only. But in this case from $(7,2) W_{\mu}^{A}(x) \equiv 0$. There is no nontrivial zero mass theory in this channel. This is consistent with the Weinberg-Witten theorem [10]. The essential observation in (12) is that the vector isotriplet mass is lowered by the contact with the second layer. From (1) we expect $m_{W} \cong O(\Lambda)$. Then from (8) $\frac{\mu^{2}}{m_{W}^{2}} \ll 1$ holds true for large enough $\Lambda^{\prime}$. In this case

$$
M_{W} \cong \mu \ll \Lambda
$$

We may view this as an effect of level repulsion opening the possibility of a large mass gap between the vector isotriplet and the other bound states contained in (1).

By varying $\mu$ we can tune the vector isotriplet mass. Therefore it is appropriate to study the phase diagram of a vector meson theory as its mass is gradually lowered.

In fact we will study the phase diagram of a compact vector meson theory. This requires the following comment.

Below we will present the lattice version of (7) as the starting point for future exact studies. The compact nature of the effective ("hadronized") fields is inherited from this lattice structure. In fact it can be shown that in the strong coupling expansion of nonabelian lattice gauge theory the physics of the bilinear hadron fields 


$$
\chi^{\dagger i}(x) \chi_{j}(x) \equiv(R(x) \cdot U(x))_{j}^{i}
$$

with $R$ and $U$ a hermitian respectively unitary matrix in SU(2) isospin space is independent of $R(x)$ so that the functional integration over the bosonic effective fields reduces to a group integration [17]. It is then reasonable to expect that also in the weak coupling regime the effective fields are compact as long as the lattice constant is nonzero. This compactness is essential for our program since the phase structure crucially depends on it [13].

Thus we study the phase structure of the theory with the following lattice action

$$
S\left[U_{\mu}, \Omega\right]=\beta \cdot \sum_{x, \mu \nu} \operatorname{tr}\left(U_{\mu}(x) U_{\nu}(x+\mu) U_{\mu}^{\dagger}(x+\nu) U_{\nu}^{\dagger}(x)\right)+K \cdot \sum_{x, \mu} \Omega^{\dagger}(x) U_{\mu}(x) \Omega(x+\mu)+\text { h.c. }
$$

For $\Omega(x)=1$ this is the straightforward lattice version of the massive spin one theory (11) $\left(U_{\mu}(x)=\exp \left(i T^{A} W_{\mu}^{A}(x)\right)\right)$. The lattice constant was put to one.

The lattice theory has two parameters, $\beta$ and $K$, which for (small lattice constant) are related to $M_{W}$ by

$$
M_{W}^{2}=\frac{K}{\beta}
$$

for fixed $\beta$ we trade then $K$ for $\mu^{2}$ in $(7,12)$.

In writing (15) we have replaced

$$
U_{\mu}(x) \rightarrow \Omega^{\dagger}(x) U_{\mu}(x) \Omega(x+\mu), \quad \Omega^{\dagger}(x) \Omega(x)=1, \Omega(x) \in S U(2), \quad \int d \Omega(x)=1
$$

so that the partition function can be written as

$$
Z=\int d \Omega d U_{\mu} e^{-S\left[U_{\mu}, \Omega\right]}
$$

This introduction of superfluous variables [13] was performed to have the formal gauge invariance

$$
U_{\mu}(x) \rightarrow \Lambda^{-1}(x) U_{\mu}(x) \Lambda(x), \quad \Omega(x) \rightarrow \Lambda^{-1}(x) \Omega(x)
$$

in (18) $(\Lambda(x)$ an element of $\mathrm{SU}(2))$. Note that the matrix $\Omega(x)$ can be parametrized by a scalar field in the fundamental representation of $S U(2)$ with unit length

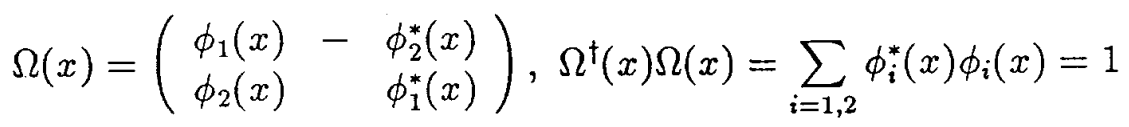


In certain regions of the parameter space $(\beta, K)(19)$ becomes a true or spontaneously broken gauge invariance as is manifest from the existence of two phases for the theory $(15,18)[11],[12],[13]:$

1. Higgs phase for large $\beta$ and $K>K_{C}(\beta)$ with

$$
<\Omega>0, \quad<U_{\mu}>\neq 0
$$

2. gauge invariant confinement phase for $K<K_{C}$ with

$$
<\Omega>=0, \quad<U_{\mu}>=0 .
$$

In the Higgs phase $M_{W} \sim<\Omega>$. At smaller $\beta$ these phases are presumably connected [12]. However in the $U(1)$ case with radial scalar field this is not so according to [11]. As in the Coleman-Weinberg mechanism [14] the scalar fields (20) have no bare mass term and we may expect a weakly first order transition (however we cannot a priori be sure that the perturbative scenario of [14] is realized on the lattice).

What concerns the order of the transition the literature [11],[12] is confusing. However in particular [18] argues for first order with the scalar field fluctuating also radially. For our purpose the transition should be weakly first order. To be precise $\langle\Omega\rangle$ should have a small nonzero jump $\Delta<\Omega>\ll \Lambda$. At this point we have reminded ourselves that (15) is embedded in the full theory (7) whose scale was. taken to be $\Lambda \cong \mathrm{O}(1 \mathrm{TeV})$. Below we comment on the viability of this scenario in our framework.

Starting then deep in the Higgs phase at large $\beta$ and $K \gg K_{c}(\beta)$ chosen such that $M_{W} \cong O(\Lambda)$ and lowering $K$ gradually towards $K_{c}(\beta)$ we move $M_{W}$ towards the critical value $M_{W}^{c} \sim \Delta<\Omega>\ll \Lambda$ which is the minimum value of $M_{W}$ in the Higgs phase.

It is this value which we identify with the weak boson mass. This is done for the following reason.

Close to the critical line the constraint $\Omega^{\dagger}(x) \Omega(x)=1$ is lifted [15],[13],[19] and $\operatorname{Tr}\left(\Omega^{\dagger}(x)\right.$ $\Omega(x)) \equiv H^{2}(x)$ develops into a full scalar field $H(x)$ which carries dimension. By (20) this is naturally identified with the Salam-Weinberg Higgs [1].

Therefore one option for the continuum limit (this limit must be taken in the full theory (7) and is discussed below) is the broken phase of a gauged $\phi^{4}$ theory (see also [19]). This scenario resembles the bosonic sector of the S.M. as far as the SU(2) of weak isospin is concerned. The $U(1)_{Y}$, respectively electromagnetism, is not yet included. Clearly $M_{W}^{c}$ sets the scale for the onset of the scalar fluctuations of $\operatorname{Tr}\left(\Omega^{\dagger}(x) \Omega(x)\right) \equiv H^{2}(x)$. Therefore the Higgs mass will be $m_{H} \cong O\left(M_{W}^{c}\right)$ and a light Higgs is obtained. This scalar mass is protected by the weakly first order character of the phase transition.

We have however idealized our case by studying (15) in isolation.

In fact the continuum limit is to be defined in the full confining theory (7) by fixing some physical mass parameter and moving towards $\beta \equiv \frac{4}{g^{2}} \rightarrow \infty, \mu \rightarrow \mu_{c}$ with $\frac{\mu}{m_{W}} \ll 1$. 
Triviality [16] of the gauged $\phi^{4}$ theory is avoided by the fact that the mass scale of the higher bound states and resonances, which is $O(\Lambda)$, acts as a physical cut-off. This raises the important possibility that the renormalized couplings in the gauge-Higgs system are weak because the cut-off is large in the sense that $M_{W}^{c} \ll O(\Lambda)$. Unlike in QCD here the low energy behaviour is not dominated by Goldstone particle interactions. So taking (15) in isolation may not be completely unrealistic. However, we expect a feature at the phase boundary which goes back to the full theory, namely that it prohibits entering the confinement phase.

Although in this phase there are no particles with open SU(2) charge the notion of confinement implies the presence of long range gauge interactions. If this phase survives in the continuum limit this is in disagreement with expectations from the Weinberg-Witten theorem [10].

In practice impenetrability of the confinement phase can occur such that the renormalized parameters $\left(\beta_{R}, K_{R}\right)$, which derive from the full theory, exclude this region. In particular the heavy mass effects will play a role in this renormalization.

Since $\langle\Omega\rangle$ decreases towards the critical line $m_{H}$ will also decrease with $K \rightarrow K_{c}$ in the Higgs phase (at this point the precise dependence is unknown). On the confinement side the "Higgs" behaves as a usual scalar, so roughly $m_{H}^{2} \approx \frac{1}{K}$.

In practical calculations (see below) this change of behaviour can serve as an indicator whether the boundary has been crossed (in fact this crossing might happen for nonzero lattice constant).

The crucial question of the present approach is obviously whether the hierarchy $M_{W}^{c} \cong$ $\mu^{c}<(<) m_{W} \cong O(\Lambda)$ is obtained at the critical line.

From [15],[19],[13] one may indeed expect that the region where $\operatorname{Tr}\left(\Omega^{\dagger}(x) \Omega(x)\right)$ starts to fluctuate as a full scalar field is substantially in the IR of the original scale $m_{W} \cong O(\Lambda)$.

This is best accounted for by blockspinning [20] or, equivalently, by integrating out the momenta in the intervall $\mu^{c} \stackrel{\sim}{<} p \leq \Lambda$. Then $<\Omega>$ should reflect as the scale of symmetry breaking the size of the scale of the unintegrated momenta $\langle\Omega\rangle \cong \mu_{c}$. The phase structure of the full theory should in fact be determined by the impenetrability of the confinement phase which assures that the $S U(2)$ of weak isospin never comes unbroken. Then $\langle\Omega\rangle \cong \mu_{c}$ is all what is needed, and the order of the phase transition becomes an empty question. It appeared only in connection with the model (15) taken as isolated from the full theory. Nevertheless if (12) gives a glimps of the right physics the Higgs phase of (15) should qualitatively reflect what goes on, namely that when a vector mass is sufficiently lowered in a description with compact variables a full Higgs phase (with radial Higgs) appears [13]. The desired behaviour $\langle\Omega>\neq 0$ at the boundary may just be a reflection of the first order character of the transition in (15). Clearly we have to put these studies on a solid quantitative basis. This is provided by the lattice regularized version of (7).

Several lattice actions which all have (7) as the naive continuum limit exist. We suggest two forms. 


$$
\begin{aligned}
S\left[\Omega, V_{\mu}, U_{\mu}, \chi\right]= & \beta \cdot \sum_{P} \operatorname{tr} U_{P}- \\
& -\frac{k}{1+\frac{b}{k}} \cdot \sum_{x, \mu}\left[\Omega^{\dagger}(x) \chi^{\dagger}(x) \sigma_{\mu} U_{\mu}(x) \chi(x+\mu) \Omega(x+\mu)-\right. \\
& \left.-\Omega^{T}(x) \chi(x) Q U_{\mu}(x) \chi(x+\mu) \Omega(x+\mu)\right]-\sum_{x} \chi(x) Q \chi(x)- \\
& -\frac{b}{1+\frac{b}{k}} \sum_{x, \mu} \operatorname{Tr}\left[V_{\mu}^{\dagger}(x) \chi^{\dagger}(x) \sigma^{\mu} U_{\mu}(x) \chi(x+\mu)\right]- \\
& +K \cdot \sum_{x, \mu} \Omega^{\dagger}(x) V_{\mu}(x) \Omega(x+\mu)+\text { h.c. }
\end{aligned}
$$

The notation is lattice standard. The first term is the gauge plaquette term with the gauge coupling $\beta$. The next three terms represent the usual fermionic lattice action written for the Weyl fermions $\chi(x)$ [5] but augmented by the $\Omega(x)$ variables. The last but one term is the lattice form of the mixing term in (7) and the last term the lattice mass term $\left(V_{\mu}(x)=\exp \left(i T^{A} V_{\mu}^{A}(x)\right)\right) . K$ trades the $\mu^{2}$ dependence in (7) (the lattice constant is one). $b$ and $k$ are hopping parameters and the factor $\left(1+\frac{b}{k}\right)^{-1}$ normalizes the kinetic term. One may give equal weight to the hopping terms and put $b=k$. However a priori $b$ and $k$ are independent. $\operatorname{Tr}$ denotes the trace in isospin space.

The partition function is

$$
Z=\int d \Omega d V_{\mu} d U_{\mu} d \chi^{\dagger} d \chi e^{-S\left[\Omega, V_{\mu}, U_{\mu}, \chi\right]}
$$

The action (23) possess the formal gauge invariance

$$
\begin{gathered}
\chi(x) \rightarrow \chi(x) \Lambda(x), \Omega(x) \rightarrow \Lambda^{-1}(x) \Omega(x), V_{\mu}(x) \rightarrow \Lambda^{\dagger}(x) V_{\mu}(x) \Lambda(x+\mu), \\
\Lambda^{\dagger}(x) \Lambda(x)=1, \Lambda(x) \in \operatorname{SU}(2) .
\end{gathered}
$$

Because $\Lambda(x) \in S U(2)$ no problems with triangle anomalies arise. In fact the invariance of the fermionic measure under (25) is essential to write the form (24) of the partition function.

With the action (23) the composite field $\chi^{\dagger}(x) \sigma_{\mu} U_{\mu}(x) T^{A} \chi(x+\mu)\left(W_{\mu}^{A}(x)\right.$ is to be considered as a limit of this field) acquires the $\Omega(x)$ variables (by $\chi(x) \rightarrow \chi(x) \Omega(x)$ ) in the same way as the variable $V_{\mu}(x)$ (last term of (23)). One may find this reasonable since both fields mix. On the other hand a simpler lattice action is obtained by unifying the fermionic kinetic term and the mixing term into one expression by writing the alternative action 


$$
\begin{aligned}
\tilde{S}\left[\Omega, V_{\mu}, U_{\mu}, \chi\right]= & \beta \cdot \sum_{P} \operatorname{tr} U_{P}-k \cdot \sum_{x, \mu}\left\{\operatorname { T r } \left[V_{\mu}^{\dagger}(x) \chi^{\dagger}(x) \sigma^{\mu} U_{\mu}(x) \chi(x+\mu]+\right.\right. \\
& \left.+\operatorname{Tr}\left[V_{\mu}^{\dagger}(x) i T_{2} \chi(x) i \sigma_{2} i \tau_{2} U_{\mu}(x) \chi(x+\mu)\right]\right\}-\sum_{x} \chi(x) Q \chi(x)- \\
& +K \cdot \sum_{x, \mu} \Omega^{\dagger}(x) V_{\mu}(x) \Omega(x+\mu)+\text { h.c. }
\end{aligned}
$$

Now there is only one hopping parameter, $k$, and when it comes to numerical simulations (26) is easier to handle than (23). Also forms intermediate between (23) and (26) can be written. However in treating $(23,26)$ numerically it seems not possible to neglect the fermion determinant because it is needed to thermalize the $V_{\mu}(x)$ variables.

Once an action of this type is given one would "measure" the correlators

$$
\begin{gathered}
\sum_{\vec{x}}<\chi^{\dagger}(0, \vec{x}) \sigma_{i} U_{i}(0, \vec{x}) T^{A} \chi(0, \vec{x}+i), \chi^{\dagger}(t, \vec{x}) \sigma_{j} U_{j}(t, \vec{x}) T^{B} \chi(t, \vec{x}+j)>\cong \\
\sum_{\vec{x}}<\chi^{\dagger}(0, \vec{x}) \sigma_{i} \chi(0, \vec{x}), \chi^{\dagger}(t, \vec{x}) \sigma_{j} \chi(t, \vec{x})>\cong \delta_{i j} e^{-M_{I=0} \cdot t} \\
\sum_{\vec{x}}<\operatorname{Tr}\left(\Omega^{\dagger}(0, \vec{x}) \Omega(0, \vec{x})\right), \operatorname{Tr}\left(\Omega^{\dagger}(t, \vec{x}) \Omega(t, \vec{x})\right)>\cong e^{-2 m_{H} \cdot t}
\end{gathered}
$$

The notation is lattice standard with $(i, j)=(1,2,3) . \quad M_{I=0}$ denotes the mass of the isosinglet $W_{\mu}(x)$ in (2).

The influence of boundary conditions is neglected in (27). One may also contemplate other interpolating operators with the right quantum numbers such as $\operatorname{Tr}\left[T^{A} V_{\mu}(x)\right]$ and $\sum_{\mu} \operatorname{Tr} V_{\mu}(x)$ in the isospin one respectively zero channel and linear combinations with those used in (27).

Tuning then $K$ to small values one should first observe that $\frac{M_{W}}{M_{I=0}}$ decreases from order one. At some critical value of $K$ the last correlator should start to develop an exponential falloff corresponding to $m_{H} \cong O\left(M_{W}\right)$ ( in the Higgs phase a constant must be substracted to see this). As mentioned, for non-zero lattice spacing a crossing into the confinement phase is likely and signaled by $m_{H}$ starting to rise with decreasing $K$. The value of $m_{H}$ should be extracted at the phase boundary (where the system should ressemble the S.M.), that is shortly before $m_{H}$ starts to rise. The ratio $\frac{m_{H}}{M_{W}}$ can then be determined.

A first test of the system may be conducted with $\Omega(x)=1$ just observing the decrease of $\frac{M_{W}}{M_{I=0}}$ as $K$ is gradually lowered.

The calculation of $m_{\text {Higgs }}$ in terms of $M_{W}$ and the eventual discovery of a light Higgs will be a crucial test for the present proposal.

A light Higgs is thus not necessarily an exclusive prediction of SUSY-GUT [21]. Moreover we expect to be able to give a concrete value of $m_{\text {Higgs }}$, not only a bound. Also we need no additional scalars as SUSY-GUT does. 
As already pointed out the $U(1)_{Y}$ of the S.M. does not appear so far in the proposal. Our approach to calculate $M_{Z} / M_{W}$ would be to add the minimal coupling to electromagnetism to $(23,26)$. Then $\chi^{\dagger} \sigma_{\mu} T^{3} \chi$ will mix with the photon.

In our case the constraint discovered in [22], namely that the weak boson be very light with respect to its radial excitation, can well be fulfilled, remember (13). Note in passing that in the bosonic sector there will be a custodial SU(2) broken only by electromagnetism.

The coupling to light fermions was completely omitted in our lattice approach. This problem is profoundly connected to the question to what extent the S.M. as we know it can really be reproduced from a lattice formulation [23].

Finally we can ask what happens to the system at nonzero temperature. The S.M. is known to pass to a symmetric phase at $T \cong 300 \mathrm{GeV}$. In our case this phase is nonexistent because the renormalization effect of the heavy resonances cannot be neglected. Therefore one approaches at this temperature the analogue of the quark-gluon plasma.

We intend to discuss the phenomenological implications of the model in the framework of an effective Lagrangian elsewhere.

\section{Acknowledgement:}

The author thanks H.B. Nielsen for discussions especially concerning [13] and F. Jegerlehner and A. Galli for reading the manuscript. He also thanks M. Locher and F. Jegerlehner for the possibility to work at the Paul Scherrer Institute and D. Wyler for support and discussion.

\section{References}

[1] S.L. Glashow, Nucl. Phys. B22 (1961) 579

S. Weinberg, Phys. Rev. Lett. 19 (1967) 1264

A. Salam, in "Elementary Particle Theory", ed. N. Svartholm (Almquist and Wiksell, Stockholm 1968) 367.

[2] H.E. Haber and G.L. Kane, Phys. Rep. C117 (1985) 75

R. Barbieri, Riv. N. Cim. 11 (1988) 1

H.P. Nilles, Phys. Rep. C110 (1984) 1.

[3] S. Weinberg, Phys. Rev. D13 (1976) 974 and Phys. Rev. D19 (1979) 1277

L. Susskind, Phys. Rev. D20 (1979) 2619

E. Farhi and L. Susskind, Phys. Rep. 74 (1981) 277

T. Appelquist, Proceedings of the Rencontre de la Vallée d'Aoste, La Thuile, Italy, 1993.

[4] T. Appelquist and C. Bernard, Phys. Rev. D22 (1980) 200

A. Longhitano, Nucl. Phys. B188 (1981) 118

J. Bagger, S. Dawson and G. Valencia, Nucl. Phys. B399 (1993) 364

R. Casalbuoni, S. De Curtis, A. Deandrea, N. Di Bartolomeo, R. Gatto, D. Dominici and F. Feruglio, Nucl. Phys. B409 (1993) 257. 
[5] H. Schlereth, Phys. Lett. B267 (1991) 513.

[6] A. Galli, Thesis (Paul Scherrer Institute), in preparation.

[7] B.W. Lee, C. Quigg and H.B. Thacker, Phys. Rev. D16 (1977) 1519

M. Chanowitz, M. Golden and H. Georgi, Phys. Rev. D36 (1987) 1490

M. Chanowitz and M.K. Gaillard, Nucl. Phys. B261 (1985) 379

J. Kuti, L. Lin and Y. Shen, Phys. Rev. Lett. 61 (1988) 678

A. Hasenfratz, K. Jansen, J. Jersàk, C.B. Lang, T. Neuhaus and H. Yoneyama, Nucl. Phys. B317 (1989) 81.

[8] H. Schlereth, Phys. Lett. B261 (1991) 463.

[9] U.-G. Meissner, Phys. Rep. 161 (1988) 213 and references therein.

[10] S. Weinberg and E. Witten, Phys. Lett. B96 (1980) 59.

[11] J. Ranft, J. Kripfganz and G. Ranft, Phys. Rev. D28 (1983) 360

T. Munehisa and Y. Munehisa, Nucl. Phys. B215 [FS7] (1983) 508

Y. Munehisa, Phys. Rev. D30 (1984) 1310

Kei-ichi Kondo, Nucl. Phys. B295 [FS21] (1988) 93.

[12] E. Fradkin and S.H. Shenker, Phys. Rev. D19 (1979) 3682

H. Kühnelt, C.B. Lang and G. Vones, Nucl. Phys. B230 [FS10] (1984) 16.

[13] D. Foerster, H.B. Nielsen and M. Ninomiya, Phys. Lett. B94 (1980) 135.

[14] S. Coleman and E. Weinberg, Phys. D7 (1973) 1888.

[15] E. Brézin and J. Zinn-Justin, Phys. Rev. B14 (1976) 3110.

[16] M. Lüscher and P. Weisz, Nucl. Phys. B290 [FS20] (1987) 25, ibid 295 [FS21] (1988) 65, ibid 318 (1989) 705 and further references therein.

[17] N. Kawamoto and J. Smit, Nucl. Phys. B192 (1981) 100.

[18] Y. Munehisa, Phys. Rev. D31 (1985) 1522.

[19] E. Brézin and J.M. Drouffe, Nucl. Phys. B200 [FS4] (1982) 93.

[20] K.G. Wilson, in "Recent Developments in Gauge Theory" eds. G.'t Hooft et al. (Plenum Press, 1980) - Particular pertinent in our context see also: H.B. Nielsen and A. Patkos, Nucl. Phys. B195 (1982) 137.

[21] JL. Lopez, D.V. Nanopoulos and A. Zichichi, CERN-TH. 6934/93, CPT-TAMU34/93, ACT-13/93 (Revised) and references therein.

[22] S. Narison, Phys. Lett. B122 (1983) 171.

[23] T. Banks and A. Dabholkar, Nucl. Phys. B (Proc. Suppl.) 29B, C (1992) 46. 
